\title{
O IMPACTO DO RELACIONAMENTO NO CONTEXTO DAS RELAÇÕES ENTRE AGÊNCIAS E CLIENTES: proposição e teste de um modelo
}

\author{
Relationship impact on the context of relations between agencies \& customers: \\ proposition and model test
}

\author{
Vinícius Sittoni Brasila, Marcelo Gattermann Perin ${ }^{\mathrm{b}}$, Cláudio Hoffmann Sampaio ${ }^{\mathrm{c}}$, \\ Max Lacherd, Ilton Teitelbaum ${ }^{\mathrm{e}}$
}

\footnotetext{
a Administrador de Empresas, Dr. Prof. do PPGAd/PUCRS. Porto Alegre, RS - Brasil, e-mail: vinicius@pucrs.br

b Administrador de Empresas, Dr. Prof. da PPGAd/PUCRS, Porto Alegre, RS - Brasil, e-mail: mperin@pucrs.br

c Administrador de Empresas, Dr. Prof. da PPGAd/PUCRS, Porto Alegre, RS - Brasil, e-mail: sampaio@pucrs.br

d Professor da ESPM, Porto Alegre, RS - Brasil, e-mail: lacher@espm.br

e Professor da FAMECOS/PUCRS, Porto Alegre, RS - Brasil, e-mail: iltontei@terra.com.br
}

\begin{abstract}
Resumo
Poucas atividades são tão adequadas para o uso do marketing de relacionamento quanto o contexto estabelecido entre agências de propaganda e seus clientes; contudo, praticamente inexistem estudos que focalizem esta questão. As características da relação estabelecida entre agências e clientes atendem a todas as premissas consideradas fundamentais para a aplicação do marketing de relacionamento, incluindo aspectos estratégicos, operacionais e de performance. Neste estudo, buscou-se explorar com maior profundidade estes aspectos. Por meio de um levantamento do tipo cross sectional e do uso da modelagem de equações estruturais, um modelo teórico é proposto e testado a partir de uma amostra de 178 grandes e médias empresas. Os resultados apontam para um impacto positivo e significativo da orientação para relacionamento por parte das agências nas avaliações feitas pelos clientes. Além disto, como se poderá notar, o desempenho de relacionamento influencia diretamente nas percepções dos clientes quanto aos resultados de mercado originados, em parte, das ações de comunicação propostas pelas agências.
\end{abstract}

Palavras-chave: Marketing de relacionamento; Performance organizacional; Agência de propaganda. 


\begin{abstract}
Few activities are as adequate for the use of relationship marketing as the established context between advertising agencies and their customers. In this sense, this study forwards to explore the characteristics of the relation between the advertising agencies and their customers, considering the fundamental premises for the relationship marketing application. Results point out to a positive and significant impact on the relationship orientation on the agency's behalf on the evaluations done by the customers. Relationship performance also has a direct influence on the customer's perception as to market results originated, in part, by the communication feats proposed by the agencies.
\end{abstract}

Keywords: Relationship marketing; Organizational performance; Advertising agencies.

\section{INTRODUÇÃO}

Relacionamentos não tornam necessariamente um cliente leal mas são fundamentais para o desenvolvimento da lealdade e, por conseqüência, para a obtenção dos benefícios dela decorrentes, tais como maior eficiência das ações de marketing e maior lucratividade por cliente, entre outros (GRÖNROOS, 2000). Ao longo das últimas duas décadas, o marketing de relacionamento assumiu um espaço de destaque no meio acadêmico, caracterizando-se por ser um campo de estudos bastante fértil e por inúmeros trabalhos fundamentais, que ecoaram entre pesquisadores e profissionais de mercado, incluindo as publicações de Berry (1983), Dwyer, Schurr e Oh (1987); Morgan e Hunt (1994); Wilson e Vlosky (1997); Grönroos (2000); Sheth e Parvatiyar (2000).

De acordo com Patterson e Ward (2000, p. 318), o "marketing de relacionamento está na linha de frente da pesquisa acadêmica e da prática do marketing", sobretudo em função das conseqüências positivas dele derivadas, com especial destaque para lealdade e maior lucratividade. Conforme Berry (2002), o marketing de relacionamento é aplicável sempre que houver uma necessidade constante de um serviço e sempre que o cliente controlar a seleção do fornecedor deste serviço, tendo alternativas de escolha.

No campo das relações estabelecidas entre agências de propaganda e clientes, o desenvolvimento de um conhecimento mais completo e profundo sobre a importância da manutenção de bons relacionamentos e de seus impactos na avaliação dos clientes, quanto aos resultados dessa relação, constitui-se em um desafio ainda pouco enfrentado na literatura de marketing. Em linhas gerais, quando se fala da avaliação dos resultados das ações de uma agência o foco concentrase principalmente em questões como eficácia da propaganda, reconhecimento e recordação (recall) e avaliação de mídias (SHIMP, 2002), havendo pouca atenção para a questão do relacionamento agênciacliente e suas conseqüências.

Dentro deste contexto, o presente artigo objetiva apresentar e testar um modelo teórico que identifique os elementos centrais do relacionamento agência-cliente, bem como de suas conseqüências nas avaliações feitas pelo cliente quanto ao desempenho da própria agência.

A estrutura metodológica do trabalho baseou-se na abordagem orientada para a descoberta (MENON et al., 1999) e buscou conciliar aspectos teóricos identificados na literatura de marketing com informações obtidas junto a profissionais de mercado, constituindo-se, assim, os construtos-base para a elaboração do modelo teórico. Posteriormente, foi desenvolvido um levantamento do tipo survey cross-sectional junto a uma amostra de grandes e médias empresas do estado do Rio Grande do Sul, a qual se constituiu a base para a validação do modelo por meio da modelagem de equações estruturais.

Neste artigo, inicialmente, é feita uma breve revisão de aspectos relevantes da teoria em marketing de relacionamento e medidas de performance de mercado. Posteriormente, o método 
de pesquisa é detalhado, apresentando as principais etapas do estudo, bem como os procedimentos de implementação e análise utilizados. Por fim, são apresentados os resultados da validação do modelo, conclusões, limitações, além das principais implicações acadêmicas e gerenciais do estudo.

\section{SÍNTESE TEÓRICA}

Antes de serem apresentados aspectos teóricos relevantes ao estudo, cabe relembrar que este é um trabalho voltado para dois pontos fundamentais: a relação entre uma empresa e sua agência de propaganda e a construção de um modelo que permita a avaliação da performance da agência a partir deste relacionamento. Desta forma, o foco da síntese teórica a seguir apresentada recai sobre estes dois pontos, muito embora, naturalmente, não esgote todas as facetas do tema.

\section{Relacionamento no contexto agência-cliente}

Conforme Patterson e Ward (2000), o termo relacionamento tem sido abordado a partir de diferentes conotações e amparado por diferentes definições. Desde a analogia entre o relacionamento de duas empresas e o casamento entre duas pessoas (LEVITT, 1985; DWYER et al., 1987; BOWEN; SIEHL; SCHNEIDER, 1989) até a abordagem baseada em modelos empíricos (MORGAN;HUNT, 1994; WETZELS; RUYTER; LEMMINK, 2000), busca-se compreender como se formam, como se mantêm e como se extinguem os relacionamentos.

Jackson (1985) já afirmava que o marketing de relacionamento é uma tarefa difícil e complexa e que não se trata da melhor escolha para todos os ofertantes ou para todo e qualquer mercado, ressaltando que os desafios de gerenciar relacionamento não podem ser ignorados. Segundo Goron e Luce (1994), "a gestão do relacionamento entre duas empresas compreende um processo organizado que lida com as atitudes e ações que conduzem a relações duradouras, visando à obtenção de benefícios mútuos, ao atingimento de objetivos compartilhados e à transferência de tecnologia."
Inúmeros autores apontam o fato de que uma adequada gestão de relacionamentos aumenta o valor da transação para o cliente, gerando vantagem competitiva para a empresa (SPEKMAN; JOHNSTON, 1986; PATTERSON; WARD, 2000; ZEITHAML; BITNER, 2003). Além disto, outros benefícios podem ser identificados como decorrência do estabelecimento de relacionamentos entre empresa e clientes (MADHAVAN et al., 1994), tais como controle, aprendizado, eficiência, estabilidade e legitimidade.

Já há algum tempo, entretanto, é reconhecido que nem sempre a opção do relacionamento de longo prazo é a melhor alternativa para todas as empresas, pois, por questões políticas ou pelas características do mercado em questão, pode ser mais vantajoso tratar cada transação como se fosse única, admitindo o leilão dos clientes (JACKSON, 1985). Diante disto, tal autora aponta as características das transações organizacionais capazes de gerar benefícios às empresas que, nelas envolvidas, buscam desenvolver relações de longo prazo. São elas: alto custo de mudança, investimentos em ativos fixos e recursos humanos, elevada importância estratégica, dependência tecnológica.

Várias dessas características podem ser observadas no contexto das relações entre agências de propaganda e seus clientes, incluindo os custos decorrentes de eventuais mudanças (não apenas os financeiros, mas também os relacionados à aprendizagem e processos) e a importância estratégica assumida pelas comunicações de marketing na grande maioria dos negócios. Neste sentido, cabe destacar as considerações de Grönroos (2000) a respeito do fato de que, embora seja externa à organização em si, uma agência de propaganda atua como parte da função ou da estrutura de marketing de uma empresa, justificando ainda mais a necessidade do estabelecimento de relações consistentes.

Além disso, mesmo em uma análise preliminar, é possível verificar que construtos tipicamente vinculados com o desenvolvimento e a manutenção de relacionamento - tais como confiança e comprometimento (MORGAN; HUNT, 1994) - são condição básica para assegurar relações duradouras entre agência e cliente. 


\section{Relacionamento e desempenho percebido da agência}

Um dos fatores fundamentais para sustentar a importância do estudo do relacionamento entre uma agência de propaganda e seus clientes é o fato de que o desenvolvimento de relacionamentos duradouros tem impacto significativo na lucratividade da primeira. Segundo Reichheld (1996), um aumento de 5\% no índice de retenção de clientes por parte de agências de propaganda repercute em ganhos de até 95\% no valor presente líquido de um cliente (ou seja, nos lucros totais durante o tempo de vida de um cliente típico).

Além disso, a percepção do relacionamento influencia as avaliações feitas pelo cliente quanto à performance do fornecedor (CANNON; PERREAULT Jr., 1999). Desta forma, pelo menos parte da avaliação feita por um cliente quanto ao desempenho de sua agência deverá estar focada nos resultados decorrentes do relacionamento estabelecido. Considerando-se as colocações de Peelan, Ekelmans e Vijn (1989), anteriormente comentadas, verifica-se que uma parte do sucesso ou fracasso do relacionamento será decorrência das decisões, ações e atitudes do próprio cliente.

Compreender melhor como se processa a avaliação do relacionamento por parte do cliente e, ainda, o impacto deste relacionamento na avaliação das ações da agência e na performance de mercado do cliente constitui-se em conhecimento fundamental para acadêmicos e práticos.

Contudo, quando se busca o que vem sendo feito no campo das comunicações de marketing, percebe-se que existe uma concentração mais focada na medição dos resultados de uma campanha, ou de anúncios em si, do que na mensuração da relação agênciacliente e de seus desdobramentos (KALRA; GOODSTEIN, 1998; ABMLER; BURNE, 1999; STEWART; FURSE, 2000).

Assim, o número de artigos de caráter acadêmico que acaba por enfatizar esta relação e as dificuldades a ela intrínsecas é bastante pequeno. A preocupação com a relação agência de propaganda-cliente surge, com certa intensidade, na década de 90 , quando se constata uma crise no setor publicitário americano. Segundo Henke (1995), em pesquisas realizadas em tal período, constatou-se que a maioria das agências norte-americanas tinha a expectativa de perder $67 \%$ de suas contas da época, considerando, para tal estimativa, um período futuro de 5 anos.

Muitas perdas de contas são atribuídas, sem a menor dúvida, tanto aos conflitos causados pelas fusões e aquisições de agências quanto às novas políticas e objetivos estabelecidos por estas, dadas as novas características do mercado, que resultam em mudanças no lado do cliente. Contudo, um número considerável de mudanças é resultado da insatisfação do cliente com a performance da agência (HENKE, 1995). Doyle, Corstjens e Michell (1980) contam que a primeira razão de mudança de agência, no Reino Unido, é a insatisfação com a performance da agência, mais do que mudanças nas políticas dos clientes e/ou das próprias agências. Usando a mesma metodologia, Michell (1986) encontrou resultados semelhantes no mercado publicitário norte-americano.

Ambos os estudos buscaram identificar quais dimensões da performance das agências foram cruciais para a mudança. Nos dois casos, a insatisfação com a "habilidade criativa" foi o fator que mais contribuiu para esta situação, enquanto que o fato de "não estar perto o suficiente" dos negócios dos clientes apareceu em segundo lugar. Esta última dimensão é nitidamente reflexo de percepções quanto à orientação para o relacionamento por parte da agência.

Outros indícios de que o desempenho do relacionamento tem sido fundamental na avaliação que os clientes fazem de suas agências são trazidos por Nicholas (1999). Em seu trabalho, este autor destaca diversas falhas identificadas junto às agências de propaganda, incluindo: falhas na oferta de serviços adequados à necessidade do cliente, dificuldades no desenvolvimento da confiança, falta de inovação, ineficiência, ausência de um controle sobre os custos e não cumprimento de promessas. O autor destaca, ainda, o fato de que o staff das agências é considerado difícil de localizar/contatar e que os responsáveis pelo planejamento e criação não compreendem adequadamente os mercados-alvo de seus clientes. Por fim, em grande escala, a maioria dos anunciantes não sente que suas agências fornecem conselhos competentes nos seus negócios e nas questões de marketing. 
Uma linha de pesquisa emergente na análise da relação das agências de propaganda com seus clientes propõem que esta relação pode ser mais produtiva e mais satisfatória para os clientes se estes forem vistos como empregados "parciais" ou participantes temporários na produção dos serviços da agência (BEARD, 1996). Esta perspectiva é amplamente reconhecida como uma das características típicas da atividade de prestação de serviços (LOVELOCK; YOUNG, 1979; MILLS; MORRIS, 1986). A natural complexidade do papel do cliente na relação discutida faz com que as agências possam ter dificuldades em satisfazê-lo se o papel por ele desempenhado for imprevisível ou inadequado (BEARD, 1996).

De certo modo, enfim, observa-se que as agências estão passando por um processo de reestruturação, fruto da necessidade de mudança no que diz respeito ao relacionamento destas com seus clientes e às conseqüências disto em sua própria performance (MADDOX, 2001).

\section{MÉTODO}

O presente estudo foi conduzido com base em três etapas centrais, a partir de uma abordagem orientada para a descoberta (MENON et al., 1999). Nesta abordagem, informações obtidas junto à literatura são complementadas por dados, informações ou insights gerados a partir de uma perspectiva baseada no mercado (entrevistas de profundidade ou grupos de discussão, por exemplo). Além disto, buscou-se desenvolver um processo de triangulação por meio do uso de procedimentos qualitativos para a geração dos construtos do modelo, de procedimentos quantitativos para a validação do modelo e, novamente, de procedimentos qualitativos para a explicação de resultados inesperados.

O método utilizado para a avaliação das relações existentes entre os construtos do modelo foi o de path analysis (HAIR Jr. et al., 1998), através da aplicação de modelagem de equações estruturais (HOYLE, 1995), seguindo-se uma estratégia de desenvolvimento de modelo (HAIR Jr. et al., 1998). Para tanto, foram considerados os dados coletados em um levantamento do tipo survey cross-sectional (CHURCHILL, 1999), realizado junto a grandes e médias empresas do estado do Rio Grande do Sul.
A modelagem de equações estruturais é usualmente empregada em estudos de investigação de relações entre construtos, tendo como base dados oriundos de levantamentos do tipo survey cross-sectional. Por meio desta técnica, o pesquisador pode investigar modelos complexos caracterizados por uma série de equações descritivas das inter-relações entre diversas variáveis endógenas e exógenas (STEENKAMP; BAUMGARTNER, 2000).

Ainda segundo Steenkamp e Baumgartner (2000), a modelagem de equações estruturais é considerada uma das mais populares e conhecidas entre as abordagens estatísticas avançadas utilizadas na área de Marketing. Quando bem aplicada, tem substancial vantagem sobre as técnicas chamadas de primeira geração, tais como análise fatorial, análise discriminante e regressão múltipla, pela grande flexibilidade que propicia ao pesquisador na ligação entre teoria e dados empíricos (CHIN, 1998).

Para Hair Jr. et al. (1998), a técnica de modelagem de equações estruturais é particularmente útil para verificar modelos teóricos mais complexos, nos quais variáveis dependentes tornam-se independentes em relações de dependência subseqüentes. Essencialmente, a modelagem de equações estruturais é uma abordagem estatística abrangente, que permite o teste de hipóteses sobre as relações entre variáveis observáveis e latentes (HOYLE, 1995).

Os detalhes dos procedimentos metodológicos realizados nesta pesquisa para a definição dos construtos do modelo, para a elaboração do instrumento de coleta e para a coleta de dados propriamente dita são apresentados a seguir.

\section{Construtos do modelo teórico e relações hipotetizadas}

Para a identificação e definição dos construtos utilizados no modelo teórico, foi desenvolvido um levantamento exploratório com base em entrevistas de profundidade e revisão da literatura. Nesta etapa o objetivo central foi identificar os fatores considerados relevantes no desenvolvimento do relacionamento entre agências e clientes e na avaliação feita pelos últimos quanto ao desempenho dos primeiros. 
Foram realizadas 22 entrevistas de profundidade junto a profissionais de agências de propaganda (incluindo as áreas de criação, planejamento, atendimento e mídia), bem como executivos de empresas-clientes, veículos e consultores atuantes no setor. Paralelamente às entrevistas, foi desenvolvido um levantamento bibliográfico, procurando-se identificar estudos anteriores focados nos aspectos de relacionamento agência-clientes e nas formas de avaliação utilizadas por estes para optarem pela manutenção ou troca de agência.

A partir dos resultados da etapa exploratória, foi elaborada uma primeira estrutura conceitual que viria a servir de base para o modelo final. Este foi composto por seis construtos: orientação para o mercado por parte do cliente (OMC), estrutura e time de apoio do cliente (ETA), suporte à tomada de decisão do cliente (STDC), operação e orientação para relacionamento por parte da agência (ORA), desempenho de mercado do cliente (DM) e desempenho do relacionamento agência-cliente (DR). Cada um dos construtos é comentado a seguir.

Orientação para o mercado por parte do cliente (OMC): a orientação para o mercado é uma das condições prévias para uma performance superior de mercado. Considerandose que as ações de uma agência de propaganda inserem-se, em última análise, nas funções de marketing de seus clientes, torna-se relevante que estes possuam um nível elevado de foco no mercado, ampliando as possibilidades de sucesso das ações da agência através de subsídios adequados em termos de informações, de uma atitude orientada para o mercado e de uma postura pró-ativa. Com base nas entrevistas de profundidade desenvolvidas, ficou evidenciado que as chances de sucesso de uma agência aumentam consideravelmente quando seus clientes possuem um nível superior de orientação para o mercado, havendo também impactos no processo de avaliação do desempenho da agência por parte do cliente. Assim:

H1) a OMC terá um impacto positivo e significante na percepção de desempenho do relacionamento com a agência;

H2) a OMC terá um impacto positivo e significante no desempenho de mercado da empresa-cliente.
Estrutura e time de apoio do cliente (ETA): um dos itens centrais no relacionamento agência-cliente refere-se à necessidade de ambas as partes disponibilizarem estruturas e equipes qualificadas em termos de comunicação, permitindo um mútuo suporte entre agência e cliente. Pelo lado da agência, tal disponibilização é condição básica e natural; contudo, pelo lado do cliente, nem sempre é visível a preocupação de oferecer à agência uma estrutura/time capaz de atender suas necessidades e de interagir e avaliar adequadamente as ações propostas. Deste modo:

H3) a ETA terá um impacto positivo e significante na percepção de desempenho do relacionamento com a agência;

H4) a ETA terá um impacto positivo e significante no desempenho de mercado da empresa-cliente.

Suporte à tomada de decisão do cliente (STDC): uma das alterações típicas na relação agência-cliente é a expectativa cada vez maior, por parte dos clientes, de que agência atue como fornecedor de informações e auxiliador no processo de tomada de decisão, demonstrando, desta forma, maior comprometimento, conhecimento e aproximação com o cliente. Assim:

H5) o STDC terá um impacto positivo e significante na percepção de desempenho do relacionamento com a agência.

Orientação para o relacionamento por parte da agência (ORA): muito embora relacionamento seja um processo que envolve pelo menos duas partes, é natural que caiba a uma delas a iniciativa pelo desenvolvimento do mesmo, assumindo uma postura capaz de incentivar a outra parte a ingressar e manter-se no relacionamento. Desta forma, a orientação para o relacionamento, por parte da agência, é condição básica para a existência de relações duradouras com seus clientes, englobando aspectos ligados à intensidade de contato, transparência, comprometimento, confiança, agilidade, integração, criatividade e planejamento. Tais elementos são antecedentes fundamentais do desempenho do relacionamento percebido pelo cliente. Neste sentido:

H6) a ORA terá um impacto positivo e significante na percepção de desempenho do relacionamento com a agência.

Desempenbo de relacionamento $(D R)$ : conforme apontado pela literatura e corroborado 
pelas entrevistas de profundidade, o relacionamento agência-cliente deve gerar resultados relevantes do ponto de vista do cliente. Assim, questões como aprendizagem, conhecimento do mercado, melhoria nos processos, ganhos de negociação e outros resultados não-financeiros são vistos como fundamentais na percepção dos clientes. O último construto incluído no modelo envolve as percepções de performance de mercado da empresa (desempenho de mercado - DM). Trata-se de um conjunto de indicadores que envolvem lembrança de marca, participação de mercado, lucratividade e resultados de vendas os quais constituem objetivos centrais de uma empresa e para os quais as ações de uma agência devem ser direcionadas. A hipótese testada é de que o impacto das ações da agência não ocorre de forma direta no desempenho de mercado do cliente, mas sim indiretamente, através da percepção deste quanto ao desempenho do relacionamento com a agência. Desta forma, a hipótese final deste estudo é:

H7) o DR terá impacto positivo e significante no desempenho de mercado da empresa-cliente.

\section{ELABORAÇÃO DO INSTRUMENTO DE COLETA}

O modelo teórico proposto exigiu a elaboração de um instrumento de coleta para a mensuração de seus construtos. Para tanto, foram utilizadas escalas disponíveis na literatura de marketing e previamente testadas em estudos empíricos. De acordo com as necessidades do trabalho, algumas escalas foram adaptadas e/ou criadas especificamente para o presente propósito.

Para operacionalização do construto OMC, utilizou-se uma escala de 8 itens adaptada de Deshpande e Farley (1999). Já para a operacionalização do construto ETA, foi desenvolvida uma escala de 6 itens. A operacionalização do construto STDC foi feita através de uma escala de 2 itens desenvolvida para este estudo. O construto ORA foi mensurado através de uma escala de 14 itens, cuja estrutura baseou-se em Wilson e Vlosky (1997), bem como em itens gerados no próprio estudo. Por fim, o construto DR foi mensurado por 8 itens gerados na etapa exploratória, enquanto que o construto DM foi mensurado a partir de uma escala de 10 itens, adaptada de Sampaio (2000). Adicionalmente, foram incluídas 7 questões relacionadas à caracterização dos entrevistados, incluindo localização, setor de atuação, abrangência geográfica de mercado, faturamento, número de funcionários, principal agência de propaganda e tempo do relacionamento com a principal agência de propaganda.

O instrumento de coleta de dados foi prétestado em um conjunto de empresas cujo perfil estava de acordo com as características anteriormente mencionadas. O pré-teste indicou homogeneidade de compreensão pelas empresas envolvidas.

\section{COLETA DE DADOS}

A coleta de dados foi desenvolvida por meio de entrevistas pessoais previamente agendadas junto aos respectivos entrevistados. Para a seleção das empresas participantes, foram contatadas grandes e médias empresas de diferentes setores, cujas matrizes estivessem estabelecidas no Rio Grande do Sul. As principais regiões de abrangência do estudo foram Grande Porto Alegre e Serra Gaúcha, muito embora tenham sido entrevistadas empresas de outras regiões.

Foram entrevistados 178 executivos de empresas de médio e grande porte. $\mathrm{O}$ requisito básico qualificador do entrevistado foi a sua efetiva participação nos processos de planejamento e nas decisões referentes à área de comunicação de suas respectivas empresas.

Assim, todos os entrevistados interagiam diretamente com a agência de propaganda de sua empresa, possuíam uma visão completa do relacionamento estabelecido e eram habilitados a avaliar os resultados deste relacionamento, bem como o desempenho de sua própria empresa.

\section{RESULTADOS DA APLICAÇÃO DA MODELAGEM DE EQƯAÇÕES ESTRUTURAIS}

Anteriormente à validação do modelo por meio da modelagem de equações estruturais, foi desenvolvida uma avaliação prévia da estrutura de fatores, a partir da análise dos componentes 
principais. Os resultados apontaram para a convergência dos itens dentro da estrutura de 6 fatores. A confiabilidade do instrumento foi testada com base no coeficiente alpha de Conbrach, cujos resultados atingiram níveis bastante satisfatórios, ainda que fosse necessária a exclusão de alguns itens para melhoria do nível de confiabilidade $(\mathrm{OMC}=0,8398$ com retenção de 7 itens; ETA $=$ 0,7121 com retenção dos 4 itens utilizados; STDC $=0,8299$ com retenção dos 2 itens originais; ORA $=0,9228$ com retenção de 13 itens; $\mathrm{DR}=0,8765$ com retenção dos 8 itens originais e $\mathrm{DM}=0,8680$ com retenção dos 10 itens originais).

A path analysis seguiu os passos recomendados por Hair Jr. et al. (1998) e Bagozzi e Edwards (1998), tendo sido necessário um procedimento de agregação total do modelo de medidas, em função da frágil relação, casos observados versus parâmetros a serem estimados no modelo inicial. Neste procedimento os construtos (variáveis latentes) de primeira ordem foram transformados em variáveis contínuas, considerando-se o somatório dos valores de seus respectivos indicadores. Destaca-se, no entanto, que o modelo de medidas simplificado permaneceu logicamente equivalente ao modelo teórico originalmente proposto. A Figura 1 apresenta o modelo de mensuração utilizado.

O exame das propriedades relativas à validade dos construtos - quais sejam, a unidimensionalidade, a confiabilidade, a validade convergente e a validade discriminante - seguiu recomendações de Garver e Mentzer (1999). Todos os construtos envolvidos no estudo demonstraram validade de construto.

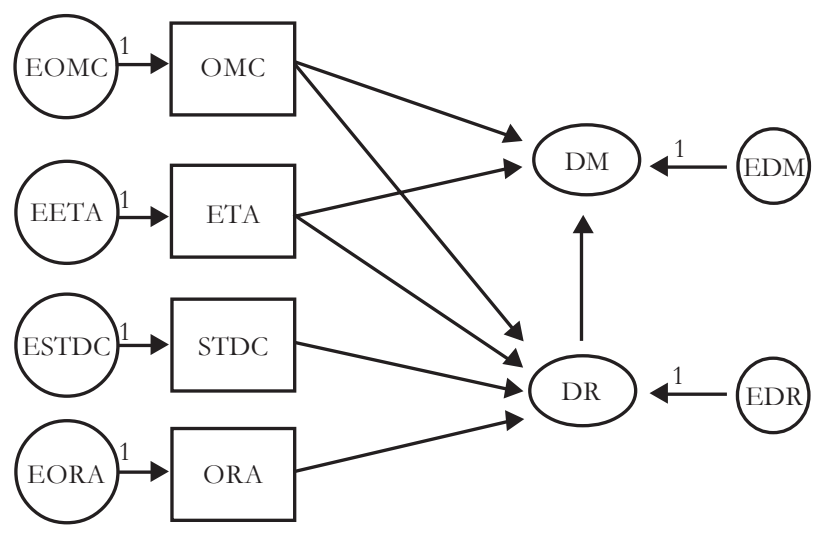

FIGURA 1 - Modelo de Mensuração
Os indicadores de estimação utilizados para a validação do modelo foram selecionados do conjunto proposto por Hair Jr. et al. (1998). Os indicadores considerados têm sido freqüentemente empregados em artigos que utilizam modelagem de equações estruturais dentro da área de Marketing (SAMPAIO, 2000; PERIN, 2002). Destaca-se que existem várias medidas de ajustamento desenvolvidas, absolutas e comparativas, não existindo, portanto, um único coeficiente que resuma o ajustamento do modelo. Além disso, não existem parâmetros rígidos no que se refere a essas medidas para aceitação ou rejeição de um modelo, dependendo de fatores como a complexidade do modelo proposto.

As medidas absolutas de ajustamento aplicadas, que determinam o grau em que o modelo geral prediz a matriz de covariância ou de correlação observada (HAIR Jr. et al., 1998), foram: Qui-quadrado sobre graus de liberdade $(\div 2$ / GL), Goodness-of-fit (GFI) e Root Mean Square Error of Approximation (RMSEA). Já as medidas comparativas de ajustamento, que comparam o modelo proposto ao modelo nulo (null mode), foram: Adjusted Goodness-of-fit (AGFI), Tucker-Lewis Index (TLI) e Comparative Fit Index (CFI).

$\mathrm{Na}$ aplicação da técnica de modelagem de equações estruturais, foram analisadas as medidas de ajustamento do modelo teórico, o nível de significância e a magnitude dos coeficientes de regressão estimados para as relações propostas pelas hipóteses do modelo.

O cálculo inicial dos índices de ajustamento apontou fortes índices de modificação em relação a duas possíveis covariâncias no modelo, quais sejam, entre o OMC e ETA e entre STDC e ORA. No sentido de identificar elementos que justificassem a inserção de tais covariâncias, optouse por analisar novamente informações obtidas a partir das entrevistas de profundidade. Do ponto de vista prático da relação entre agência e cliente, a intensidade da orientação para o mercado do cliente será manifestada, em grande parte, pela existência de uma estrutura e de um time de apoio capazes de interagir adequadamente com a agência.

Com relação à covariância entre STDC e ORA, evidencia-se que a orientação para relacionamento da agência determina uma maior aproximação com o cliente, resultando em uma participação mais efetiva no processo de tomada 
de decisão, principalmente na condição de fornecedor de informações que subsidiem as decisões do cliente.

Neste sentido, optou-se por inserir no modelo as relações de associação supracitadas. O novo cálculo de índices de ajustamento demonstrou uma boa adequação do modelo teórico, de acordo com o exame das medidas de ajustamento nela expressas. O teste de Qui-Quadrado, na sua relação com os graus de liberdade, manifestou valor aceitável $(2,145)$. O RMSEA esteve dentro dos padrões de ajustamento $(0,080)$. As demais medidas $(\mathrm{GFI}=0,973$; $\mathrm{AGFI}=0,920 ;$ TLI $=$ $0,914$; $\mathrm{CFI}=0,960)$ foram todas superiores a 0,9 registrando o ajustamento do modelo testado.

O exame da significância e magnitude dos parâmetros estimados (cargas fatoriais padronizadas) permitiu a verificação das hipóteses estabelecidas no modelo teórico, uma vez confirmado o seu ajustamento. Em termos gerais, a identificação da significância de um coeficiente de regressão (parâmetro estimado) é realizada pela análise do seu respectivo t-value. Valores de t-values superiores a 1,96 definem um nível de significância de, no mínimo, 0,05 (DUNN; SEAKER; WALLER, 1994; GARVER; MENTZER, 1999). A constatação de um coeficiente de regressão significativo implica a consideração de que a relação entre as duas variáveis se comprova empiricamente (HAIR Jr. et al., 1998) e, no caso da avaliação positiva ou satisfatória das medidas de ajustamento, destaca a validade preditiva do modelo (GARVER; MENTZER, 1999).

A Figura 2 apresenta o modelo de mensuração ajustado, bem como relaciona os parâmetros estimados. Note-se que todas as relações hipotetizadas se mostraram positivas e significativas ( $t$-value $>1,96)$. Por sua vez, a análise das cargas fatoriais, que representam a intensidade de cada relação, registrou duas relações mais frágeis no modelo, ou seja, o impacto positivo de ETA em DM $(\mathrm{g}=0,149, \mathrm{p}<0,05)$ e de ETA em DR $(g=0,180, p<0,05)$.

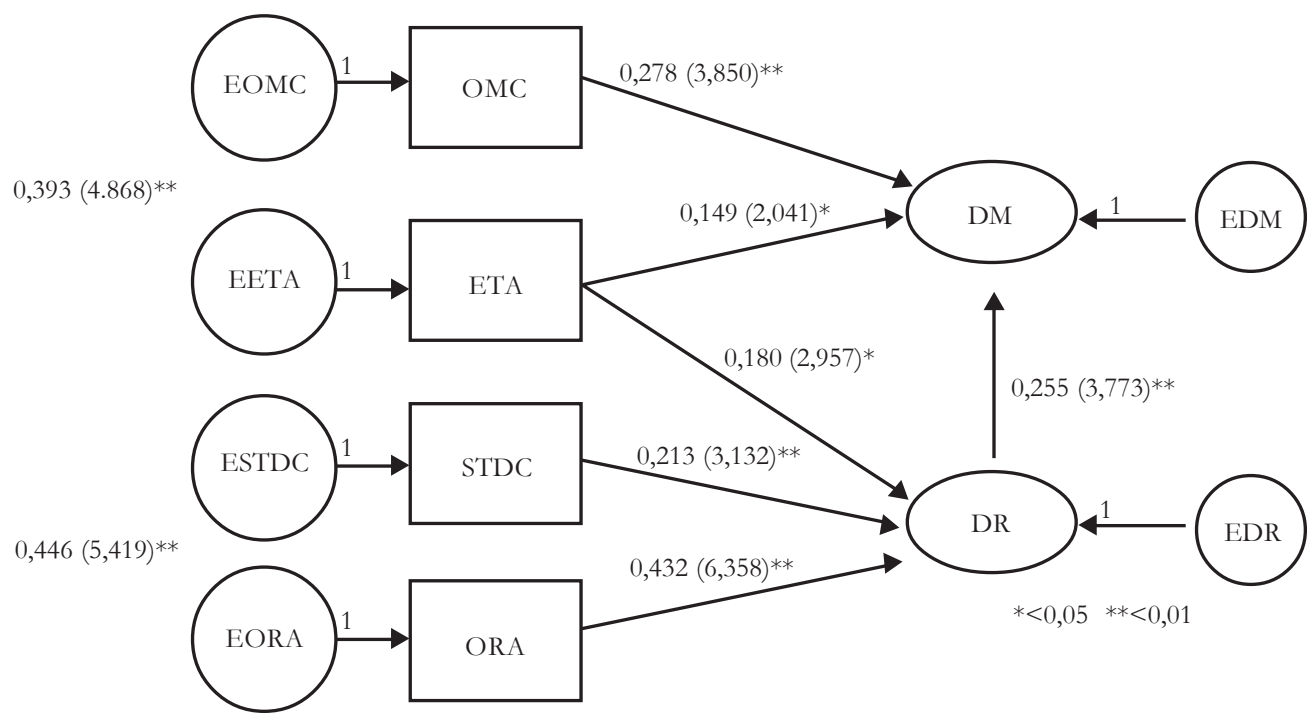

FIGURA 2 - Parâmetros Estimados do Modelo de Mensuração Ajustado (hipóteses confirmadas)

Com o intuito de aprofundar os resultados identificados com a path analysis, verificou-se a correlação entre os construtos do modelo. Esta técnica estatística permite a verificação da existência de associação entre duas variáveis, demonstrando o grau de impacto que uma delas exerce sobre a outra - e vice-versa (MALHOTRA, 2001). A Tabela 1 apresenta as correlações entre os referidos indicadores. 
TABELA 1 - Correlação entre os Construtos do Modelo

\begin{tabular}{llllll}
\hline & ORA & ETA & STDC & OMC & DM \\
\hline ETA & 0,105 & & & & \\
STDC & $0,446^{* *}$ & 0,094 & & & \\
OMC & $0,230^{* *}$ & $0,393^{* *}$ & $0,183^{* *}$ & & \\
DM & $0,263^{* *}$ & $0,315^{* *}$ & $0,210^{* *}$ & $0,405^{* *}$ & \\
DR & $0,539^{* *}$ & $0,242^{* *}$ & $0,417^{* *}$ & $0,296^{* *}$ & $0,369^{* *}$ \\
\hline
\end{tabular}

Note-se que existe uma correlação, não manifesta no modelo teórico, entre STDC e OMC, além da correlação entre ORA e OMC. Este resultado pode ser explicado a partir da perspectiva de Grönroos (2000), o qual aponta as ações de uma agência como parte integrante das funções de marketing das empresas-clientes. Assim, o apoio ao processo de tomada de decisão do cliente, bem como a orientação da agência no sentido de interagir e integrar-se fortemente ao negócio do cliente, constituem elementos correlacionados com a orientação para mercado por parte do cliente.

Em síntese, os resultados encontrados representaram bons índices de ajustamento, conforme padrões sugeridos por Hair Jr. et al. (1998), indicando que o modelo teórico testado pode ser considerado como estatisticamente válido. A partir disto, as conclusões, limitações e principais implicações decorrentes dos resultados encontrados no presente estudo são discutidas a seguir.

\section{CONCLUSÕES, IMPLICAÇÕES E LIMITAÇÕES DO ESTUDO}

Este estudo procurou explorar uma das áreas mais propensas à implementação do marketing de relacionamento, qual seja o contexto das relações estabelecidas entre as agências de propaganda e seus clientes. O gerenciamento das comunicações de marketing de uma empresa é um dos elementos centrais na função de marketing, sendo, contudo, geralmente desempenhado por um parceiro externo (GRÖNROOS, 2000). Tal fato amplia a necessidade e a importância de agências e clientes desenvolverem mecanismos capazes de aproximar as duas partes, melhorando o desempenho das ações de ambas.

Há nítidas evidências que apontam para o desempenho do relacionamento com a agência como um dos fatores centrais da avaliação feita pelo cliente quanto às ações de seu parceiro. Deste modo, o relacionamento assume não apenas um papel-chave dentro dos processos estabelecidos entre agência-cliente, mas também no que se refere à avaliação deste quanto aos resultados obtidos a partir da relação com a agência.

Com base nos resultados encontrados no estudo, serão, a seguir, as apresentadas as principais conclusões, implicações para futuros estudos e limitações do trabalho.

Inicialmente, cabe destacar que 6 das 7 hipóteses levantadas no modelo teórico foram confirmadas. O modelo de mensuração demonstrou impactos positivos e significativos de OMC em DM; de ETA em DR e DM; de STDC em DR e de ORA em DR; além da relação entre DR e DM. Esta última, sem dúvida, é uma das principais contribuições do presente trabalho.

Em linha com a busca de modelos capazes de auxiliar a mensuração da eficácia das ações de uma agência junto a seus clientes, o modelo testado caracteriza-se como uma abordagem inicial ao tema. Ao identificar-se a relação positiva e significativa entre o desempenho do relacionamento e o desempenho de mercado, fica evidenciado que as ações da agência serão percebidas pelo cliente de forma indireta. Ou seja, uma melhor avaliação do relacionamento resulta em um impacto positivo no desempenho de mercado percebido pelo cliente, um dos objetivos centrais das ações de comunicação de marketing.

Os resultados encontrados demonstram, também, que a melhoria dos resultados de mercado do cliente depende de uma estreita relação estabelecida entre este e sua agência. Mais especificamente, a orientação para relacionamento por parte da agência (ORA) caracteriza-se como o principal antecedente da avaliação de desempenho do relacionamento, seguido de outro construto correlacionado ao primeiro (STDC). Com relação 
a este último construto, fica nítida a importância de as agências assumirem um papel de fornecedores de informações, oferecendo um suporte adequado à tomada de decisão de seus clientes. Tais resultados repercutem na forma como as agências devem gerenciar o relacionamento com seus clientes, apontando para a necessidade de "estar perto o suficiente" para reduzir as insatisfações decorrentes de um eventual distanciamento, conforme apontado por Michell (1986).

Observa-se que a orientação para o relacionamento por parte da agência atende aos quatro níveis de retenção apresentados por Berry e Parasuraman (1992) e Zeithaml e Bitner (2003). Do ponto de vista dos laços financeiros (nível 1), o relacionamento proporciona aos clientes ganhos financeiros resultantes não apenas da redução de custos ou preços especiais, mas também a partir da negociação realizada pela agência junto a terceiros.

O segundo nível de retenção é caracterizado pelos laços sociais, ou seja, aspectos mais voltados aos relacionamentos interpessoais. Questões relacionadas com relacionamentos interpessoais e afinidade com o atendimento da agência emergiram como um dos resultados mais relevantes no momento de o cliente avaliar o relacionamento com a agência. Neste sentido, os resultados encontrados no presente trabalho vão ao encontro dos obtidos por Wetzels, Ruyter e Lemmink (2000), os quais identificaram que as características do relacionamento em mercados business-to-business impactam positivamente no comprometimento afetivo entre as partes. Este, por sua vez, impacta positivamente nas intenções de lealdade por parte dos clientes.

O terceiro nível de retenção caracterizase pelos laços de customização, os quais implicam em um conhecimento mais íntimo do cliente, possibilitando o desenvolvimento de soluções individualizadas e inovadoras, bem como a antecipação de necessidades do cliente. No trabalho de Nicholas (1999), comentado anteriormente, um dos pontos mais criticados por parte dos clientes é exatamente a ausência desta intimidade que resulta em soluções pouco inovadoras por parte das agências e no desconhecimento das características e necessidades do mercado-alvo dos clientes.

Por fim, o quarto nível de retenção aponta para a formação de laços estruturais. Embora mais complexos de serem implementados, a proposta de integrar sistemas de informações e compartilhar processos (tais como a própria geração de informação e desenvolvimento conjunto de pesquisas de mercado) caracteriza o ponto mais elevado do relacionamento entre agências e clientes, guardando nítida relação com aspectos de relacionamento e de suporte à tomada de decisão do cliente, discutidos anteriormente.

Assim, a melhoria da percepção do impacto das ações das agências na performance de mercado de seus clientes pode ser obtida por meio de uma efetiva orientação para o relacionamento por parte da agência.

Outro resultado relevante que decorre do presente estudo refere-se ao impacto positivo da orientação para mercado por parte do cliente (OMC) em seu desempenho de mercado (DM). Esta relação já foi identificada dentro do contexto brasileiro em diversos estudos anteriormente realizados (SAMPAIO, 2000; PERIN, 2002). À medida que o desempenho de mercado é, em última análise, o principal indicador de sucesso das ações de uma agência e que ele é diretamente relacionado com o nível de orientação para o mercado por parte do cliente, identifica-se uma oportunidade de ampliação do escopo de ação das agências. Em outras palavras, a avaliação das ações da agência pelo cliente poderá ser positivamente influenciada na medida em que a agência auxilie o desenvolvimento da orientação para o mercado de seus clientes.

No que se refere às implicações para futuras pesquisas, observam-se algumas considerações relevantes. A análise dos resultados da aplicação da modelagem de equações estruturais demonstrou que todas as hipóteses assumidas no estudo foram confirmadas; entretanto, a identificação de correlações não previstas no modelo teórico demonstra que há espaço para estudos orientados a uma melhor definição dos construtos aqui utilizados.

Ainda no que tange a futuras pesquisas, seria pertinente explorar e agregar outros construtos capazes de melhorar a capacidade preditiva do modelo no que se refere à influência das ações da agência no desempenho de mercado de seus clientes. Estudos nesta linha contribuiriam significativamente para a ampliação do entendimento desta relação, bem como atenderiam aos chamamentos de caráter acadêmico e prático relacionados com o desenvolvimento de métricas aplicadas às ações de marketing. 
Em termos de limitações, podem ser destacados pelo menos dois aspectos específicos. Primeiro, diante da especificidade do foco do estudo e da ausência de outros estudos similares, podem ser observadas limitações referentes ao escopo teórico abordado neste artigo. Estas limitações foram, em grande parte, contornadas por meio da abordagem metodológica utilizada, a qual procurou complementar as evidências teóricas com uma perspectiva obtida junto ao mercado.

Uma segunda limitação refere-se ao escopo geográfico do estudo, o qual se concentrou em empresas-clientes cujas matrizes situam-se no estado do Rio Grande do Sul. As características do mercado gaúcho em termos de relacionamentos agência-clientes podem não se repetir em outras regiões, implicando a necessidade de estudos focados em outros mercados a fim de comprar os resultados obtidos, identificando características peculiares de cada região.

\section{REFERÊNCIAS}

AMBLER, T.; BURNE, T. The impact of affect on memory of advertising. Journal of Advertising Research, v. 39, n. 2, p. 25-34, 1999.

BAGOZZI, R. P.; EDWARDS, J. R. A general approach for representing constructs in organizational research. Organizational

Research Methods, v. 1, n. 1, p. 45-87, Jan. 1998.

BEARD, F. Integrated marketing communications: new role expectations and performance issues in the client-ad agency relationship. Journal of Business Research, v. 37, n. 3, p. 207-216, 1996.

BERRY, L. Relationship marketing. In: BERRY, L.; SHOSTACK, L.; UPAH, G. Emerging perspectives on marketing services. Chicago: American Marketing Association, 1983.

Relationship marketing of services: perspectives from 1983 and 2000. Journal of Relationship Marketing, v. 1, n. 1, p. 9-18, 2002.

BERRY, L.; PARASURAMAN, A. Serviços de marketing: competindo através da qualidade. São Paulo: Maltese-Norma, 1992.
BOWEN, D. E.; SIEHL, C.; SCHNEIDER, B. A framework for analyzing customer service orientation in manufacturing. Academy of Management Review, v. 14. n. 1, p. 75-95, 1989.

CANNON, J.; PERREAULT Jr., W. Buyer-seller relationship in business markets. Journal of Marketing Research, v. 36, p. 439-460, 1999.

CHIN, W. W. Issues and opinion on structural equation modeling. MIS Quarterly, v. 22, n. 1, p. 7-15, Mar. 1998.

CHURCHILL Jr., G. A. Marketing research: methodological foundations. Orlando: The Dryden Press, 1999.

DESHPANDE, R.; FARLEY, J. Market orientation: summary scale. In: BEARDEN, W.; NETEMEYER, R. Handbook of marketing scales. $2^{\text {nd }}$ ed. Thousand Oaks: Sage, 1999.

DOYLE, P.; JENS, M. C.; MICHELL, P. Signals of vulnerability in agency-client relations. Journal of Marketing, v. 40, n. 4, p. 18-23, 1980.

DUNN, S. C.; SEAKER, R. F.; WALLER M. A. Latent variables in business logistic research: scale development and validation. Journal of Business Logistics, v. 15, n. 2, p. 145-172, 1994.

DWYER, F. R.; SCHURR, P.; OH, S. Developing buyer-seller relationships. Journal of Marketing, v. 51, n. 2, p. 11-27, 1987.

GARVER, M. S.; MENTZER, J. T. Logistics research methods: employing structural equation modeling to test for construct validity. Journal of Business Logistics, v. 20, n. 1, p. 33-57, 1999.

GORON, F.; LUCE, F. Gestão de relacionamento em marketing de serviços. In: ENCONTRO ANUAL DA ASSOCIAÇÃO NACIONAL DE PÓS-GRADUAÇÃO E PESQUISA EM ADMINISTRAÇÃ O, 18., 1994, Curitiba. Anais... Curitiba, 1994. p. 205-215. v. 7.

GRÖNROOS, C. Service management and marketing: a customer relationship management approach. Chinchester: Wiley, 2000. 
HAIR Jr., J. F. et al. Multivariate data analysis. $5^{\text {th }}$ ed. New Jersey: Prentice Hall, 1998.

HENKE, L. L. A longitudinal analysis of the ad agency relationship. Journal of Advertising Research, v. 35, n. 2, p. 24-30, 1995.

JACKSON, B. B. Winning and keeping industrial customers. Lexington: Lexington Books, 1985.

HOYLE, R. H. (Ed.). Structural equation modeling: concepts, issues, and applications. London: SAGE, 1995.

KALRA, A.; GOODSTEIN, R. The impact of advertising position strategies on consumer price sensitivity. Journal of Marketing Research, v. 35, p. 210-224, 1998.

LEVITT, T. A imaginação de marketing. São Paulo: Atlas, 1985.

LOVELOCK, C. H.; YOUNG, R. Look to consumer to increase productivity. Harvard Business Review, v. 57, n. 3, p. 168-178, 1979.

MADHAVAN, R.; SHAH, R. H.; GROVER, R. Motivations for and theoretical foundations of relationship marketing. In: OF 1994 AMA WINTER EDUCATORS' CONFERENCE. 1994, Chicago. Proceedings... Chicago, 1994. p. 183-190.

MADDOX, K. Reinventing the ad agency. Bto-B, v. 86, n. 9, p. 21-30, Apr. 2001.

MICHELL, P. C. Auditing of agency-client relations. Journal of Advertising Research, v. 26, n. 6, p. 29-41, 1986.

MALHOTRA, N. K. Pesquisa de marketing: uma orientação aplicada. Porto Alegre: Bookman, 2001.

MENON, A. et al. Antecedents and consequences of marketing strategy making: a model and a test. Journal of Marketing, v. 63, n. 2, p. 18-40, 1999.

MILLS, P. K.; MORRIS, J. Client as 'partial' employees of service organization: role development in client participation. Academy of Management Review, v. 11, n. 4, p 726-735, 1986.
MORGAN, R.; HUNT, S. The commitmenttrust theory of relationship marketing. Journal of Marketing, v. 58, p. 20-38, 1994.

NICHOLAS, R. Survey finds ad agencies still failing clients. Marketing, v. 11, n. 4, p. 103130, June 1999.

PATTERSON, P.; WARD, T. Relationship marketing and management. In: SWARTZ, T.; IACOBUCCI, D. Handbook of services marketing and management. Thousand Oaks: Sage, 2000.

PEELAN, P.; EKELMANS, E.; VIJN, V. Client role ambiguity and satisfaction in clientad agency relationship. Journal of Advertising Research, v. 39, n. 5, p. 69-78, Mar./Apr. 1989.

PERIN, M. G. A relação entre orientação para mercado, aprendizagem organizacional e performance. 2002. $181 \mathrm{f}$. Tese (Doutorado em Administração) - PPGA / Escola de Administração, UFRGS, Porto Alegre, 2002.

REICHHELD, F. A estratégia da lealdade. Rio de Janeiro: Campus, 1996.

SAMPAIO, C. H. Relação entre orientação para o mercado e performance empresarial em empresas de varejo de vestuário do Brasil. 2000. 220 f. Tese (Doutorado em Administração) - PPGA / Escola de Administração, UFRGS, Porto Alegre, 2000.

SHETH, J.; PARVATIYAR, A. Handbook of relationship marketing. Thousand Oaks: Sage, 2000.

SHIMP, T. Propaganda e promoção: aspectos complementares da comunicação integrada de marketing. Porto Alegre: Bookman, 2002.

SPEKMAN, R. E.; JOHNSTON, W. J. Relationship management: managing the selling and buying interface. Journal of Business Research, v. 14, n. 7, p. 519-531, 1986.

STEENKAMP, J. E. M.; BAUMGARTNER, $\mathrm{H}$. On the use of structural equation models for Marketing modeling. International Journal of Research in Marketing, v. 17, n. 2/3, p. 195-202, Sept. 2000. 
STEWART, D.; FURSE, D. Analysis of the impact of executional factor on advertising performance. Journal of Advertising Research, v. 26, n. 6, p. 85-88, Nov./Dec. 2000.

WETZELS, M.; RUYTER, K.; LEMMINK, J. Antecedents and consequences of service quality in business-to-business services. In: SWARTZ, T.; IACOBUCCI, D. Handbook of services marketing and management. Thousand Oaks: Sage, 2000.

WILSON, E.; VLOSKY, R. Partnering relationship activities: building theory from case study research. Journal of Business Research, v. 39, n. 9, p. 59-70, 1997.

ZEITHAML, V.; BITNER, M. J. Marketing de serviços: a empresa com foco no cliente. Porto Alegre: Bookman, 2003.

Recebido: 03/12/2007

Received: 12/03/2007

Aprovado: 15/12/2007

Approved: 12/15/2007 